CERN-TH.7526/94

BI-TP 63/94

\title{
QUARKONIUM PRODUCTION IN HADRONIC COLLISIONS
}

\author{
R. Gavai ${ }^{1}$, D. Kharzeev ${ }^{2,3}$, H. Satz ${ }^{2,3}$ \\ G. A. Schuler ${ }^{2}$, K. Sridhar ${ }^{2}$, R. Vogt $^{4}$
}

\begin{abstract}
:
We summarize the theoretical description of charmonium and bottonium production in hadronic collisions and compare it to the available data from hadron-nucleon interactions. With the parameters of the theory established by these data, we obtain predictions for quarkonium production at RHIC and LHC energies.
\end{abstract}

To appear in

Hard Processes in Hadronic Interactions

H. Satz and X.-N. Wang (Editors)

1 Tata Institute of Fundamental Research, Bombay 400 005, India

2 Theory Division, CERN, CH-1211 Geneva 23, Switzerland

3 Fakultät für Physik, Universität Bielefeld, D-33501 Bielefeld, Germany

4 Nuclear Science Division, LBL, University of California, Berkeley CA 94720, USA

CERN-TH.7526/94

BI-TP $63 / 94$

December 1994 
The production of quarkonium states below the open charm/bottom thresholds presents a particular challenge to QCD. Because of the relatively large quark masses, $c \bar{c}$ and $b \bar{b}$ production should be perturbatively calculable. However, the subsequent transition from the predominantly colour octet $Q \bar{Q}$ pairs to physical quarkonium states can introduce nonperturbative aspects. These may lead to some model-dependence, requiring cross checks with as much data as possible.

A generalisation of the colour evaporation model [1-5] provides a unified approach to the production of the different quarkonium states below the open charm/bottom thresholds. As a specific example, we consider charmonium production, although all arguments apply to bottonium production as well. Parton-parton interactions lead to the production of $c \bar{c}$ pairs, as shown in Fig. 1. We calculate the total "hidden" charm cross section, $\tilde{\sigma}_{c \bar{c}}$, by integrating over the $c \bar{c}$ pair mass from $2 m_{c}$ to $2 m_{D}$. At high energy, the dominant production mechanism is gluon fusion (Fig. 1a), so that

$$
\tilde{\sigma}_{c \bar{c}}(s)=\int_{4 m_{c}^{2}}^{4 m_{D}^{2}} d \hat{s} \int d x_{1} d x_{2} g\left(x_{1}\right) g\left(x_{2}\right) \sigma(\hat{s}) \delta\left(\hat{s}-x_{1} x_{2} s\right),
$$

with $g(x)$ denoting the gluon density and $\sigma$ the $g g \rightarrow c \bar{c}$ cross section. In pion-nucleon collisions, there are also significant quark-antiquark contributions (Fig. 1b), which become dominant at low energies. Subsequently, the $c \bar{c}$ pair neutralizes its colour by interaction with the collision-induced colour field ("colour evaporation"). During this process, the $c$ and the $\bar{c}$ either combine with light quarks to produce charmed mesons, or they bind with each other to form a charmonium state. More than half of the subthreshold cross section $\tilde{\sigma}_{c \bar{c}}$ in fact goes into open charm production (assuming $m_{c} \lesssim 1.5$ $\mathrm{GeV}$ ); the additional energy needed to produce charmed hadrons is obtained (in general nonperturbatively) from the colour field in the interaction region. The yield of all charmonium states below the $D \bar{D}$ threshold is thus only a part of the total sub-threshold cross section: in this aspect the model we consider is a generalisation of the original colour evaporation model [1-5], which neglected the contribution of $\tilde{\sigma}_{\bar{c} \bar{c}}$ to open charm production. Using duality arguments, it equated $\tilde{\sigma}_{c \bar{c}}$ to the sum over the charmonium states below the $D \bar{D}$ threshold.

Neither the division of $\tilde{\sigma}_{c \bar{c}}$ into open charm and charmonia nor the relative charmonium production rates are specified by the generalised colour evaporation model. Hence its essential prediction is that the energy dependence of charmonium production is that of $\tilde{\sigma}_{c \bar{c}}(s)$. As a consequence, the ratios of different charmonium production cross sections are energy-independent. In Fig. 2, we show the ratio of $J / \psi$ production from the decay $\chi_{c} \rightarrow \gamma J / \psi$ to the total $J / \psi$ production rate [5,6]. It provides a measure of the $\chi_{c} /(J / \psi)$ rate and is seen to be independent of incident energy for both pion and proton beams. In Fig. 3, we show the measured $\psi^{\prime} /(J / \psi)$ ratio $[5,7,8]$; it is also found to be independent of the incident energy, as well as of the projectile (pion or proton) and target (from protons to the heaviest nuclei [9]). Moreover, it is noteworthy that the ratio $\psi^{\prime} /(J / \psi)$ measured at high transverse momenta at the Tevatron [10] is quite compatible with the $p_{T}$-integrated fixed target and ISR data (Fig. 4).

The available bottonium data [11-14] also agree with constant production ratios, as seen in Fig. 5 for the ratios $\Upsilon^{\prime} / \Upsilon$ and $\Upsilon " / \Upsilon$ up to Tevatron energies. 
The present data thus support one essential prediction of the colour evaporation model up to $1.8 \mathrm{TeV}$. We now check if it also correctly reproduces the variation of the production cross sections with incident energy in this region. In Figs. 6 and 7 we show the energy dependence of $J / \psi$ production in $p N$ collisions,

$$
\sigma_{p N \rightarrow J / \psi}(s)=f_{J / \psi}^{p} \tilde{\sigma}_{c \bar{c}}(s),
$$

as obtained from the hidden charm cross section $\tilde{\sigma}_{c \bar{c}}$ calculated in next-to-leading order [15] and with the normalisation $f_{J / \psi}^{p}$ fixed empirically. We have used the MRS D-' [16] and GRV HO [17] parametrisations of the nucleon parton distributions functions [18]. For the GRV set, we have used $m_{c}=1.3 \mathrm{GeV}$, with both renormalisation and factorisation scales fixed to $m_{c}$. In the MRS calculation, $m_{c}=1.2 \mathrm{GeV}$ was used, with the scales set at $2 m_{c}$. These parameters provide an adequate description of open charm production, although the results tend to lie somewhat below the measured total $c \bar{c}$ cross sections [19]. In Figs. 6 and 7 we show only the MRS D-' result; the GRV HO result differs by less than $5 \%$ in this energy range. The agreement with the data [5] over the entire range is quite satisfactory, with the normalisation $f_{J / \psi}^{p}=0.025$. In Fig. 8 we find equally good agreement for the energy dependence of $J / \psi$ production with pion beams. However, the fraction of $J / \psi$ in the hidden charm cross section must be slightly higher to reproduce the pion data well, with $f_{J / \psi}^{\pi}=0.034$ for a good fit. This may well be due to greater uncertainties in the pionic parton distribution functions. We have also calculated the leading order cross section; the resulting theoretical K-factor, $K \equiv \tilde{\sigma}_{c \bar{c}}^{N L O} / \tilde{\sigma}_{c \bar{c}}^{L O}$, remains between 2.0 and 2.5 over the currently measured energy range for both sets of parton distribution functions and for pion and proton beams.

The fraction of $\tilde{\sigma}_{c \bar{c}}$ producing charmonium rather than open charm is thus about 10\%. This is in accord with open charm calculations, which show [19] that much of the total cross section comes from subthreshold $c \bar{c}$ initial states which acquire the necessary energy for $D \bar{D}$ formation from the interaction colour field. To illustrate this, Fig. 9 shows the fraction of the total open charm cross section with $2 m_{c} \leq M \leq 2 M_{D}$, where $M \equiv \sqrt{\hat{s}}$. It remains quite large even at very high incident energies.

We further compare the longitudinal momentum dependence of charmonium production with recent experimental results. In fig. 10 we compare data with our calculations for the $x_{F}$ dependence of $J / \psi$ production at several energies and for $\pi-p, \bar{p}-p$ and $p-p$ collisions [20]. Since there is a spread of integrated cross section values around the average $\tilde{\sigma}_{c \bar{c}}$, as seen in Figs. $6-8$, we have normalised the calculated $x_{F}$ distribution to the integrated experimental one. We conclude that the $x_{F}$ distributions are also consistent with the colour evaporation model.

Next we comment briefly on the transverse momentum distributions. We are interested in low $p_{T}$ charmonium production, for which the model provides essentially no prediction. There is the intrinsic transverse momentum of the initial partons, the intrinsic momentum fluctuations of the colour field which neutralises the colour of the $c \bar{c}$ system in the evaporation process, and at larger $p_{T}$ higher order perturbative terms. Since there is no way to separate these different contributions in the low $p_{T}$ region, the model has no predictive power. 
The colour evaporation model thus reproduces correctly both the energy dependence and the $x_{F}$ distributions of charmonium production, up to an open normalisation constant for each charmonium state, which can be fixed empirically by data. Once this is done, integrated and differential cross sections can be predicted for RHIC and LHC energies. From the fits to the data shown in Figs. 6 and 7 we obtain

$$
\left(\frac{d \sigma_{p N \rightarrow J / \psi}}{d y}\right)_{y=0}=2.5 \times 10^{-2}\left(\frac{d \tilde{\sigma}_{c \bar{c}}^{N L O}}{d y}\right)_{y=0}
$$

for $J / \psi$ production. In Fig. 11 we show the resulting $\left(d \sigma_{p N \rightarrow J / \psi} / d y\right)_{y=0}$ as function of the center of mass energy, $\sqrt{s}$, and in Fig. 12 we give the rapidity distributions at RHIC and LHC energies. The cross sections are listed in Table 1.

Before commenting on our predictions, we first repeat the analysis for $\Upsilon$ production. Because the data generally give the sum of $\Upsilon, \Upsilon^{\prime}$ and $\Upsilon$ " production, the branching ratios cannot simply be removed. Therefore we show in Fig. 13 the measured cross section for the sum of the three $\Upsilon$ states in the dilepton decay channel, denoted by $B(d \sigma / d y)_{y=0}$. We see that

$$
B\left(\frac{d \sigma_{p N \rightarrow \Upsilon}}{d y}\right)_{y=0}=1.6 \times 10^{-3}\left(\frac{d \sigma_{b \bar{b}}^{N L O}}{d y}\right)_{y=0}
$$

gives a good description of the data up to and including ISR results. The results are also calculated using the MRS D-' and GRV HO parton distribution functions, with $m_{b}=4.75 \mathrm{GeV}$ and the scales equal to $m_{b}$. Assuming the bulk of the cross section to be from $\Upsilon(1 \mathrm{~S})$ production, and using the corresponding branching ratio, we estimate from eq. (4) that about $7 \%$ of the sub-threshold $b \bar{b}$ cross section leads to $\Upsilon$ production.

Using the normalisaton determined in Eq. (4), we obtain the cross section for high energy $\Upsilon$ production; the results are shown in Figs. 14 and 15 and in Table 2 . The recent high energy data from UA1 [21] and CDF [14] agree very well with the predicted energy dependence, as seen in Fig. 13, giving strong support to the "new" parton distribution functions based on HERA data [22]. They also give us considerable confidence in the extrapolation to LHC energy.

We now comment on some features of our predictions. The two parton distribution functions, MRS D-' and GRV HO, provide fully compatible results in the measured energy range. The $\Upsilon$ predictions agree with data even up to energies close to the LHC range. The MRS D-' $J / \psi$ cross section is about twice as large as the GRV HO prediction at LHC energies. This is because the MRS distributions require larger factorisation scales than the GRV distributions. Both parton distribution functions, with their chosen scales, also give acceptable fits to the measured open charm production cross sections (see [19] for more details). The difference thus gives some indication of the uncertainty of the $J / \psi$ prediction. The $\Upsilon$ results agree over the entire energy range, since $m_{b}=4.75$ $\mathrm{GeV}$ was used as the scale in both sets.

At LHC energies, both the $J / \psi$ and $\Upsilon$ rapidity distributions remain rather constant out to $y \simeq 4$, using the MRS parton distributions. The GRV HO results show an even 
wider plateau. In either case, there is a large window for forward detection at high energies. At RHIC energies, the $J / \psi$ distributions are not as broad, with a forward plateau of 2 - 3 units for the MRS set, while the GRV distributions are somewhat narrower. The $\Upsilon$ rapidity distributions at RHIC energies are quite similar for both sets.

Finally we note that the cross sections calculated with the recent parton distribution functions are considerably higher, typically by about a factor 20, than those given by an earlier empirical parametrisation, $\sim \exp (-15 M / \sqrt{s})$, labelled CR in Figs. 11 and 14 [23-25] . This increase, confirmed by new high energy $\Upsilon$ data (Fig. 14), is mainly due to the increase in the gluon distribution functions at small $x$, as suggested by data from HERA [22].

The colour evaporation model addresses the common energy behaviour of the different quarkonium states. To determine their relative production rates, the colour evaporation process has to be specified in more detail. Let us consider one example of this. Assume that the initial colour octet state first neutralises its colour by interaction with the surrounding colour field, producing a colour singlet $c \bar{c}$ state. The relative weights of $J / \psi$ and $\psi^{\prime}$ production can then be expressed [5] in terms of the corresponding masses and the squared charmonium wave functions at the origin,

$$
\frac{\sigma\left(\psi^{\prime}\right)}{\sigma(\psi)}=\frac{R_{\psi^{\prime}}^{2}(0)}{R_{\psi}^{2}(0)}\left(\frac{M_{\psi}}{M_{\psi^{\prime}}}\right)^{5} .
$$

Here $\psi$ denotes the directly produced $1 \mathrm{~S} c \bar{c}$ state, in contrast to the experimentally observed $J / \psi, 40 \%$ of which originates from radiative $\chi_{c}$ decays (see Fig. 1). The wave functions at the origin can in turn be related to the dilepton decay widths $\Gamma_{e e} \sim$ $\left(R^{2}(0) / M^{2}\right)[5]$, giving

$$
\frac{\sigma\left(\psi^{\prime}\right)}{\sigma(\psi)}=\frac{\Gamma_{\psi^{\prime}}}{\Gamma_{\psi}}\left(\frac{M_{\psi}}{M_{\psi^{\prime}}}\right)^{3} .
$$

Inserting the measured masses and decay widths, we find

$$
\frac{\sigma\left(\psi^{\prime}\right)}{\sigma(\psi)} \simeq 0.24
$$

To compare this to the measured value of $\sigma\left(\psi^{\prime}\right) / \sigma(\psi)$, we have to remove the $\chi_{c}$ contributions from the experimental ratio,

$$
\frac{\sigma\left(\psi^{\prime}\right)}{\sigma(\psi)}=\left[\frac{1}{1-\left(\sigma_{\chi_{c}} / \sigma_{J / \psi}\right)}\right]\left[\frac{\sigma\left(\psi^{\prime}\right)}{\sigma(J / \psi)}\right]_{\mathrm{exp}} .
$$

With the experimental values $\sigma\left(\psi^{\prime}\right) / \sigma(J / \psi) \simeq 0.14$ (see Figs. 2 and 3) and $\left(\sigma_{\chi_{c}} / \sigma_{J / \psi}\right) \simeq$ 0.4 (see Fig. 1), this yields $\sigma\left(\psi^{\prime}\right) / \sigma(\psi) \simeq 0.23$, in good agreement with the theoretical result (7). We thus find that the projection of the colour singlet $c \bar{c}$ state onto $J / \psi$ and $\psi^{\prime}$ correctly describes their production ratios at all energies and transverse momenta.

The predictions for direct bottonium production ratios corresponding to eq. (7) are

$$
\frac{\sigma\left(\Upsilon^{\prime}\right)}{\sigma(\Upsilon)} \simeq 0.36 \quad ; \quad \frac{\sigma\left(\Upsilon^{\prime \prime}\right)}{\sigma(\Upsilon)} \simeq 0.27
$$


Since the contributions from indirect production through radiative $\chi_{b}$ decay are not yet known and there is also feeding from higher S-states, a quantitative comparison is not possible here. Nevertheless, the predicted values differ from the data (see Fig. 5) by less than $50 \%$ and hence appear reasonable.

So far, the most complete description of the colour evaporation process is attempted in the colour singlet model [26], in which not only the $c \bar{c}$ formation but also the subsequent colour neutralisation is assumed to take place on a perturbative scale. The resonance formation is then determined by the appropriate wave functions with the right quantum numbers, as above. As a result, the production cross section for each charmonium state is completely determined to the order of perturbation theory used. Some characteristic production diagrams in lowest order are illustrated in Fig. 16. As generally formulated, the scale of the strong coupling constant in all perturbative diagrams is determined by the mass of the heavy quark.

Such a perturbative description of colour neutralisation can be justified only if all momentum scales are sufficiently large. However, as seen in Fig. 16, colour neutralisation for all but $\eta_{c}, \chi_{0}$ and and $\chi_{2}$ requires the emission or absorption of a "third" gluon. This restricts the possible applicability of the model to production at large transverse momentum. In the $p_{T}$-integration, the "third" gluon is soft $\left(k \sim \Lambda_{\mathrm{QCD}}\right)$ in a significant part of phase space, and hence the model becomes unreliable here even though the integration is infrared finite. It is therefore not surprising that the colour singlet model leads to charmonium production ratios which disagree rather strongly with experiment. The quantum numbers of the $\chi_{2}$ allow partonic production at order $\alpha_{s}^{2}$, while $J / \psi, \chi_{1}$ and $\psi^{\prime}$ production are all of order $\alpha_{s}^{3}$. As a result, their production is much too strongly suppressed in comparison to the $\chi_{2}$. Thus, while the model predicts $\chi_{2} /(J / \psi) \simeq 10$, the measured ratios are below $2[6]$. The inclusion of certain relativistic corrections can somewhat reduce this discrepancy [5]. - Similar arguments hold for $\Upsilon$ production, although the soft part of the $p_{T}$-integration is relatively smaller, so that here the predictions may be closer to the data.

For the validity of a perturbative treatment, the "third" gluon has to be hard enough to resolve the $c \bar{c}$ into individual quarks. Hence its momentum must be higher than $1 / r_{J / \psi} \simeq 1 /(0.2 \mathrm{fm}) \simeq 1 \mathrm{GeV}$. Below this limit, it is not clear how colour neutralisation is achieved; presumably nonperturbative interactions of the colour octet $c \bar{c}$ with the gluon condensate play a considerable role. Some additional contributions can perhaps also be obtained by summing classes of perturbative contributions [27]. However, as long as the additional interactions cannot be determined quantitatively, the prediction of the $p_{T}$-integrated production ratios of the different charmonium states is not possible. It is not known if this is also true for bottonium production, or if here the role of soft processes has become sufficiently reduced to allow a fully perturbative treatment. 


\section{References:}

1) M. B. Einhorn and S. D. Ellis, Phys. Rev. D12 (1975) 2007.

2) H. Fritzsch, Phys. Lett. 67B (1977) 217.

3) M. Glück, J. F. Owens and E. Reya, Phys. Rev. D17 (1978) 2324.

4) J. Babcock, D. Sivers and S. Wolfram, Phys. Rev. D18 (1978) 162.

5) For a recent review, see G. A. Schuler, "Quarkonium Production and Decays", CERN Preprint CERN-TH.7170/94, February 1994.

6) L. Antoniazzi et al., Phys. Rev. Lett. 70 (1993) 383.

7) L. Antoniazzi et al., Phys. Rev. D46 (1992) 4828.

8) C. Lourenco, " $J / \psi, \psi^{\prime}$ and Dimuon Production in $p-A$ and $S-U$ Collisions at $200 \mathrm{GeV} /$ Nucleon", Dissertation, Technical University of Lisbon, Portugal.

9) B. Ronceux, Nucl. Phys. A566 (1994) 371c.

10) The CDF Collaboration, " $J / \psi, \psi^{\prime} \rightarrow \mu^{+} \mu^{-}$and $B \rightarrow J / \psi, \psi^{\prime}$ Cross Sections", Fermilab Preprint Fermilab-Conf-94/136-E, May 1994 (Contribution to the International Conference on High Energy Physics, Glasgow, Scotland 1994);

E. Braaten et al., Phys. Lett. B333 (1994) 548.

11) K. Ueno et al., Phys. Rev. Lett. 42 (1979) 486.

12) T. Yoshida et al., Phys. Rev. D 39 (1989) 3516.

13) G. Moreno et al., Phys. Rev. D 43 (1991) 2815.

14) V. Papadimitriou (CDF), " $\Upsilon$ Production at CDF", Fermilab Preprint FermilabConf-94-221-E, August 1994.

15) M. L. Mangano, P. Nason and G. Ridolfi, Nucl. Phys. B405 (1993) 507.

16) A. D. Martin, R. G. Roberts and W. J. Stirling, Phys. Lett. B 306 (1993) 145, and "Structure Functions and Parton Distributions, in this volume.

17) M. Glück, E. Reya and A. Vogt, Z. Phys. C53 (1993) 127.

18) H. Plothow-Besch, Comp. Phys. Comm. 75 (1993) 396, and "PDFLIB", in this volume.

19) P. L. McGaughey et al., "Heavy Quark Production in $p p$ Collisons", in this volume.

20) C. Akerlof et al., Phys. Rev. D48 (1993) 5067;

L. Antoniazzi et al., Phys. Rev. D46 (1992) 4828;

M. S. Kowitt et al., Phys. Rev. Lett. 72 (1994) 1318;

C. Biino et al., Phys. Rev. Lett. 58 (1987) 2523;

V. Abramov et al., "Properties of $J / \psi$ production in $\pi^{-}-B e$ and $p-B e$ Collisions at $530 \mathrm{GeV} / \mathrm{c} "$, Fermilab Preprint FERMILAB-PUB-91/62-E.

21) K. Eggert and A. Morsch (UA1), private communication.

22) M. Derrick et al. (Zeus), Phys. Lett. B 316 (1993) 412;

I. Abt et al. (H1), Nucl. Phys. B 407 (1993) 515.

23) N. Craigie, Phys. Rep. 47 (1978) 1.

24) R.Vogt, Atomic Data and Nuclear Data Tables 50 (1992) 343.

25) H. Satz, Nucl. Phys. A544 (1992) 371c. 
26) C. H. Chang, Nucl. Phys. B 172 (1980) 425;

E. L. Berger and D. Jones, Phys. Rev. D 23 (1981) 1521;

R. Baier and R. Rückl, Phys. Lett. B 102 (1981) 364 and Z. Phys. C 19 (1983) 251.

27) M. Beneke and V. M. Braun, "Naive Non-Abelianisation and Resummation of Fermion Bubble Chains", DESY Preprint DESY 94-200, November 1994;

M. Neubert, "Scale Setting in QCD and the Momentum Flow in Feynman Diagrams", CERN Preprint CERN-TH.7487/94, December 1994. 
Table 1: $J / \psi$ Production

\begin{tabular}{|r|r|r|}
\hline$\sqrt{s} \quad[\mathrm{GeV}]$ & $(d \sigma / d y)_{y=0}^{\mathrm{MRS}} \quad[\mu \mathrm{b}]$ & $(d \sigma / d y)_{y=0}^{\mathrm{GRV}} \quad[\mu \mathrm{b}]$ \\
\hline & $6.2 \times 10^{-2}$ & $5.8 \times 10^{-2}$ \\
20 & $1.6 \times 10^{-1}$ & $1.5 \times 10^{-1}$ \\
40 & $2.5 \times 10^{-1}$ & $2.4 \times 10^{-1}$ \\
60 & $3.5 \times 10^{-1}$ & $3.4 \times 10^{-1}$ \\
100 & $6.3 \times 10^{-1}$ & $5.9 \times 10^{-1}$ \\
200 & $1.5 \times 10^{+0}$ & $1.2 \times 10^{+0}$ \\
500 & $3.2 \times 10^{+0}$ & $2.5 \times 10^{+0}$ \\
1000 & $1.6 \times 10^{+1}$ & $5.9 \times 10^{+0}$ \\
5500 & $4.1 \times 10^{+1}$ & $1.1 \times 10^{+1}$ \\
14000 & & \\
& &
\end{tabular}

Table 2: $\left(\Upsilon+\Upsilon^{\prime}+\Upsilon^{\prime \prime}\right)$ Production

\begin{tabular}{|r|r|r|}
\hline$\sqrt{s} \quad[\mathrm{GeV}]$ & $(B d \sigma / d y)_{y=0}^{\mathrm{MRS}} \quad[\mathrm{pb}]$ & $(B d \sigma / d y)_{y=0}^{\mathrm{GRV}} \quad[\mathrm{pb}]$ \\
\hline & $3.1 \times 10^{-4}$ & $2.5 \times 10^{-4}$ \\
15 & $9.7 \times 10^{-1}$ & $9.7 \times 10^{-1}$ \\
30 & $1.2 \times 10^{+1}$ & $1.2 \times 10^{+1}$ \\
60 & $3.4 \times 10^{+1}$ & $3.7 \times 10^{+1}$ \\
100 & $8.6 \times 10^{+1}$ & $1.0 \times 10^{+2}$ \\
200 & $2.5 \times 10^{+2}$ & $3.4 \times 10^{+2}$ \\
500 & $5.5 \times 10^{+2}$ & $8.8 \times 10^{+2}$ \\
1000 & $3.0 \times 10^{+3}$ & $3.6 \times 10^{+3}$ \\
5500 & $7.8 \times 10^{+3}$ & $7.7 \times 10^{+3}$ \\
14000 & & \\
\end{tabular}




\section{Figure Captions}

Fig. 1: Lowest order $c \bar{c}$ production through gluon fusion (a) and quark-antiquark annihilation (b).

Fig. 2: The ratio of $\left(\chi_{1}+\chi_{2}\right) \rightarrow J / \psi$ to total $J / \psi$ production, as a function of the center of mass energy, $\sqrt{s}$, by proton (open symbols) and pion beams (solid symbols) [6].

Fig. 3a: The ratio of $\psi^{\prime}$ to $J / \psi$ production as a function of the center of mass energy, $\sqrt{s}$, on proton (circles) and nuclear targets (squares) $[5,7,8]$. The average value is $0.14 \pm 0.03$.

Fig. 3b: The ratio of $\psi^{\prime}$ to $J / \psi$ production by proton beams as a function of the atomic mass number $A$ for data in the energy range $20 \leq \sqrt{s} \leq 40 \mathrm{GeV}$ [9]. The average value is $0.14 \pm 0.01$.

Fig. 4a: The ratio of $\psi^{\prime}$ to $J / \psi$ production as a function of transverse momentum [10]; the shaded strip shows the average value of Fig. 3 .

Fig. $4 \mathrm{~b}$ : The ratio of $\psi^{\prime}$ to $J / \psi$ production as a function of center of mass energy, $\sqrt{s}$. The fixed target and ISR data are integrated over the low $p_{T}$ region, while the CDF point is the average over $5 \leq p_{T} \leq 15 \mathrm{GeV}$.

Fig. 5: The ratios of $\Upsilon$ ' and $\Upsilon$ " to $\Upsilon$ production as a function of the center of mass energy, $\sqrt{s}[11-14]$. The average values are $0.53 \pm 0.13$ and $0.17 \pm 0.06$, respectively.

Fig. 6: The differential $J / \psi$ production cross section $\left(d \sigma_{J / \psi}^{p N} / d y\right)=2.5 \times 10^{-2}\left(d \tilde{\sigma}_{c \bar{c}}^{p N} / d y\right)$ at $y=0$, calculated with MRS D-' parton distributions, compared to data [5].

Fig. 7: The $J / \psi$ production cross section $\sigma_{J / \psi}^{p N}=2.5 \times 10^{-2} \tilde{\sigma}_{c \bar{c}}^{p N}$ for $x_{F}>0$, calculated with MRS D-' parton distributions, compared to data [5].

Fig. 8: The $J / \psi$ production cross section $\sigma_{J / \psi}^{\pi N}=3.4 \times 10^{-2} \tilde{\sigma}_{c \bar{c}}^{\pi N}$ for $x_{F}>0$, calculated with MRS D-'/SMRS P2 parton distributions, compared to data [5].

Fig. 9: The fraction of the total open charm cross section due to the "hidden" charm mass interval $\left[2 m_{c}, 2 m_{D}\right]$.

Fig. 10a: The $J / \psi$ longitudinal momentum distributions compared to $\bar{p} N$ and $p N$ data [20], with $x_{F}=p_{L}(J / \psi) / p_{\max }(J / \psi)$; the MRS results are denoted by a solid, the GRV by a dashed line.

Fig. 10b: The $J / \psi$ longitudinal momentum distributions compared to $\pi N$ data [20], with $x_{F}=p_{L}(J / \psi) / p_{\max }(J / \psi)$. the MRS results are denoted by a solid, the GRV by a dashed line.

Fig. 11: Energy dependence of $\left(d \sigma_{J / \psi}^{p N} / d y\right)_{y=0}$ for $J / \psi$ production, as obtained with MRS D-' and GRV HO parton distributions.

Fig. 12: Rapidity distributions for $J / \psi$ production, calculated with MRS D-' parton distributions at RHIC and LHC energies. 
Fig. 13: The differential $\Upsilon$ production cross section $\left(d \sigma_{\Upsilon}^{p N} / d y\right)=1.8 \times 10^{-3}\left(d \sigma_{b \bar{b}}^{p N} / d y\right)$ at $y=0$, calculated with MRS D-' parton distributions, compared to data [5]. The corresponding GRV HO predictions are very similar.

Fig. 14: Energy dependence of $\left(d \sigma_{\Upsilon}^{p N} / d y\right)_{y=0}$ for $\Upsilon$ production, with high energy data from $[21,14]$; the predictions of MRS D-' and GRV HO essentially coincide. Also shown $(\mathrm{CR})$ is the phenomenological fit of [23].

Fig. 15: Rapidity distributions for $\Upsilon$ production calculated with MRS D-' parton distributions at RHIC and LHC energies.

Fig. 16: Lowest order contributions to charmonium production in the colour singlet model. 

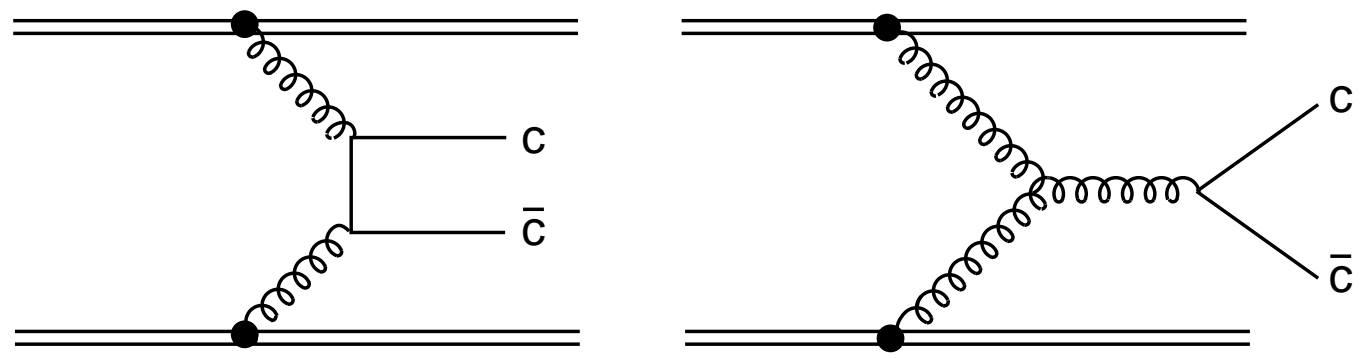

(1a)

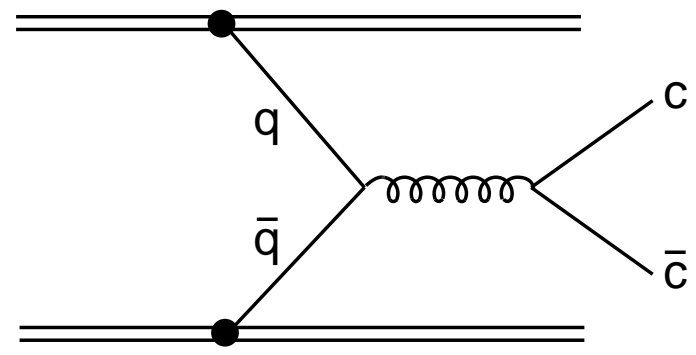

(1b)

Figure 1: 


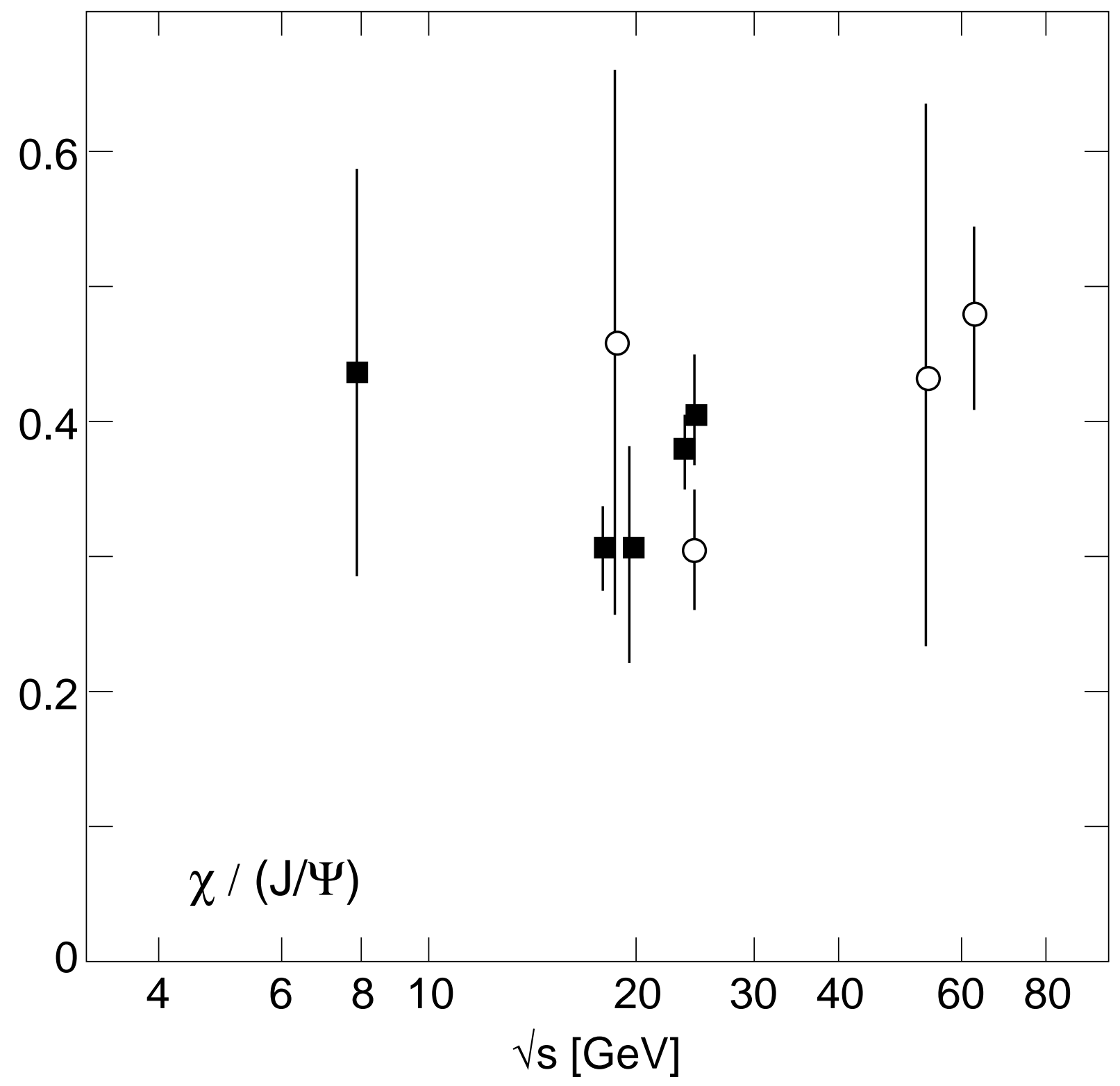

Figure 2: 

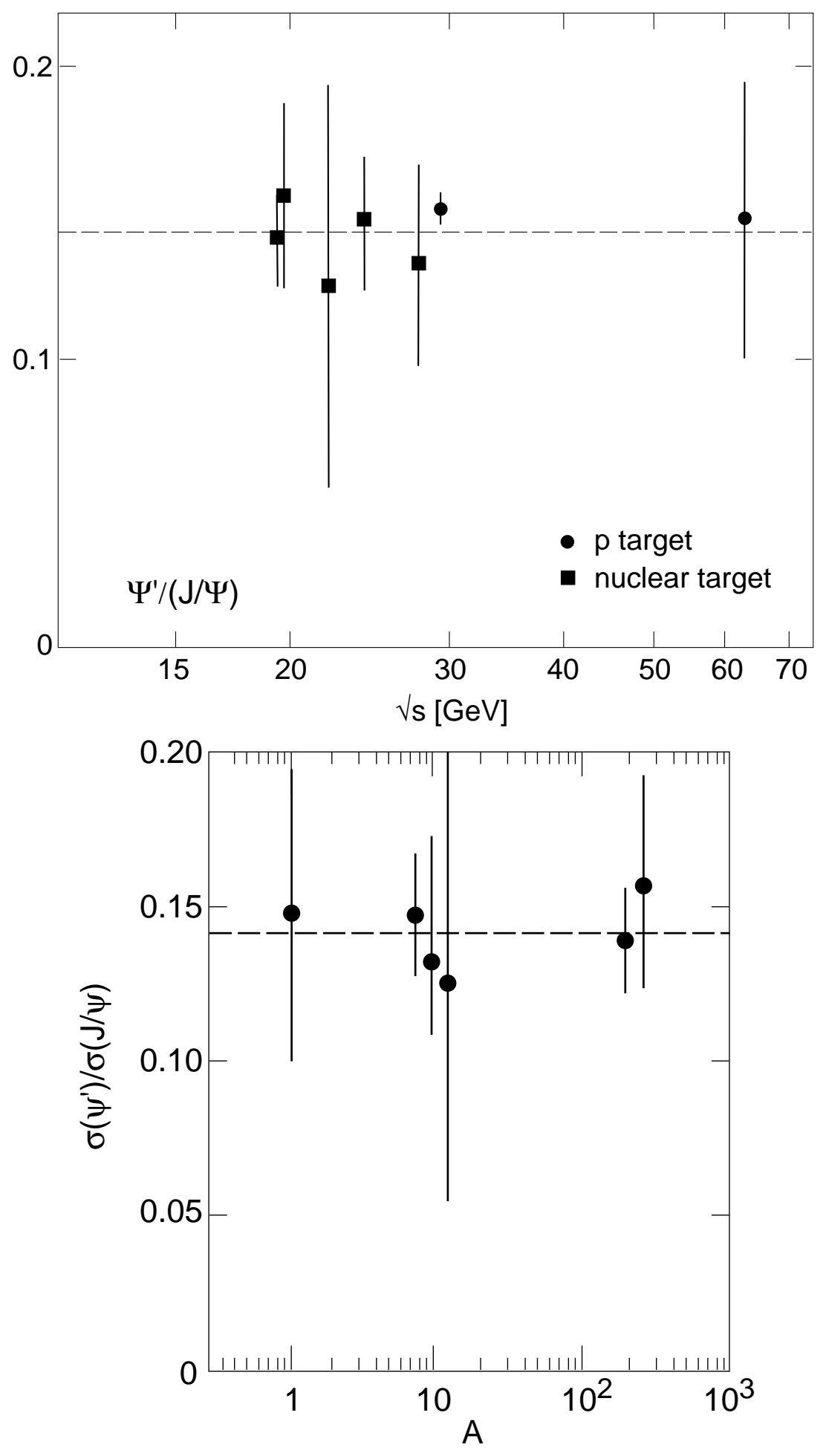

Figure 3: 

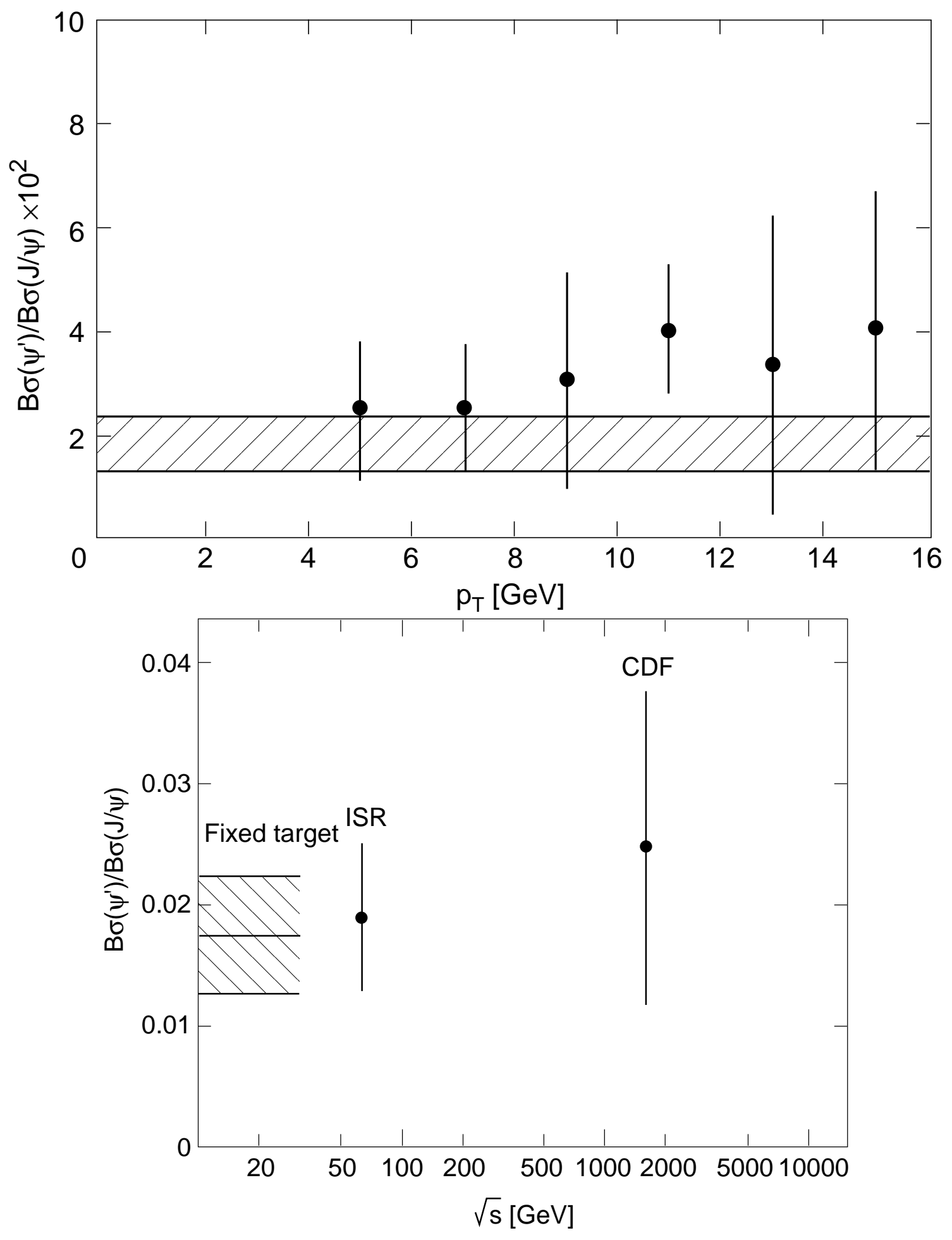

Figure 4: 


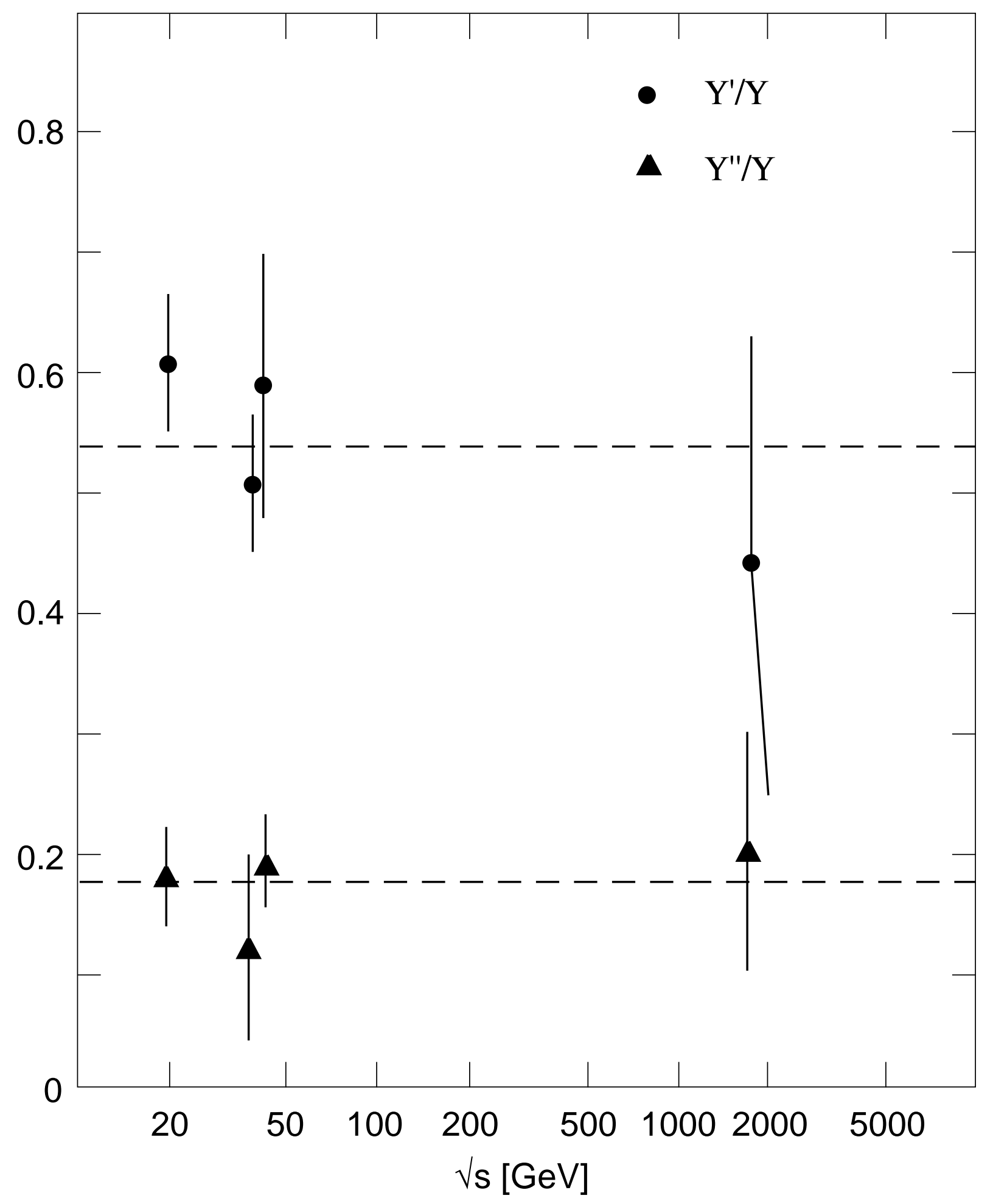

Figure 5: 


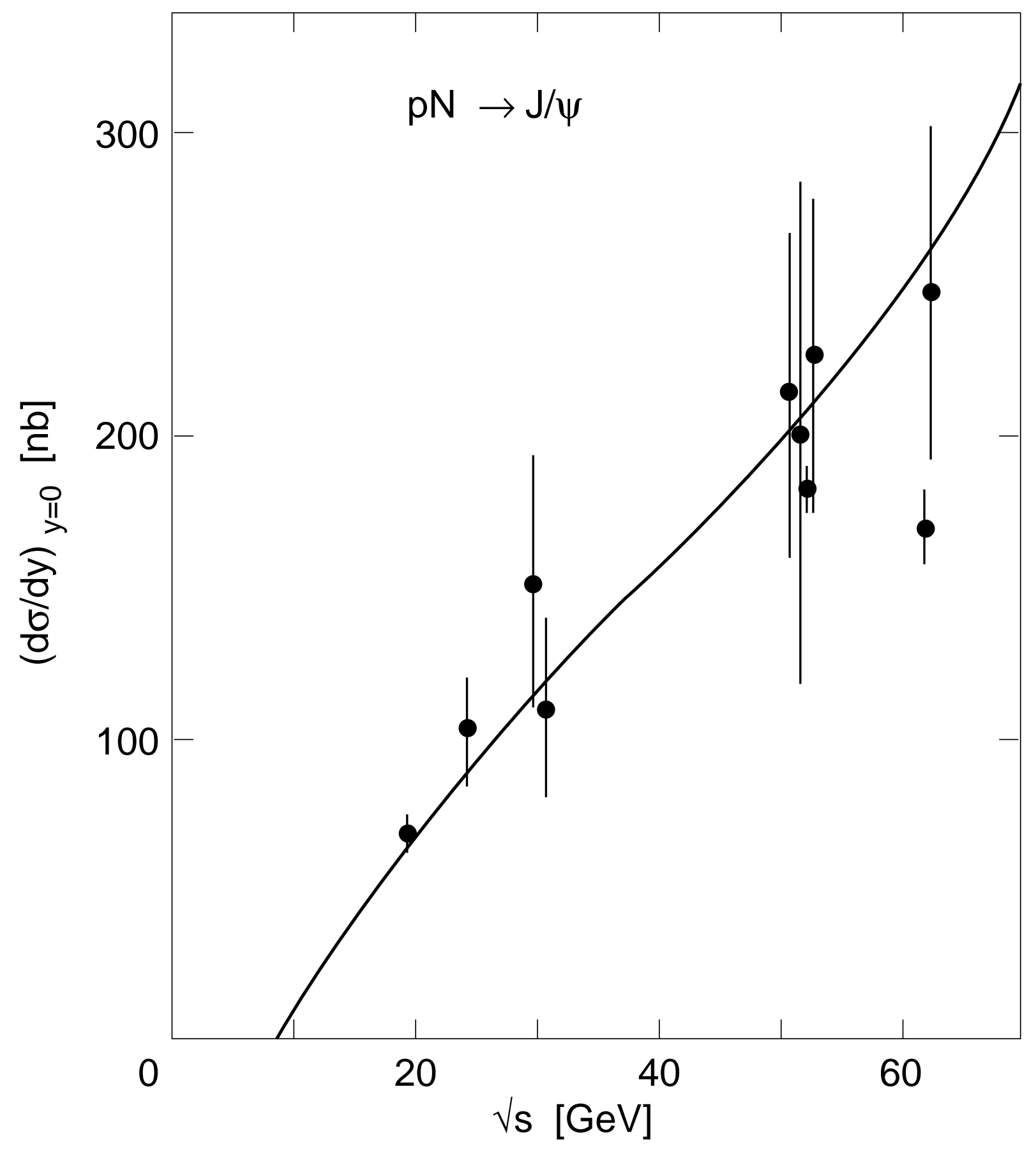

Figure 6: 


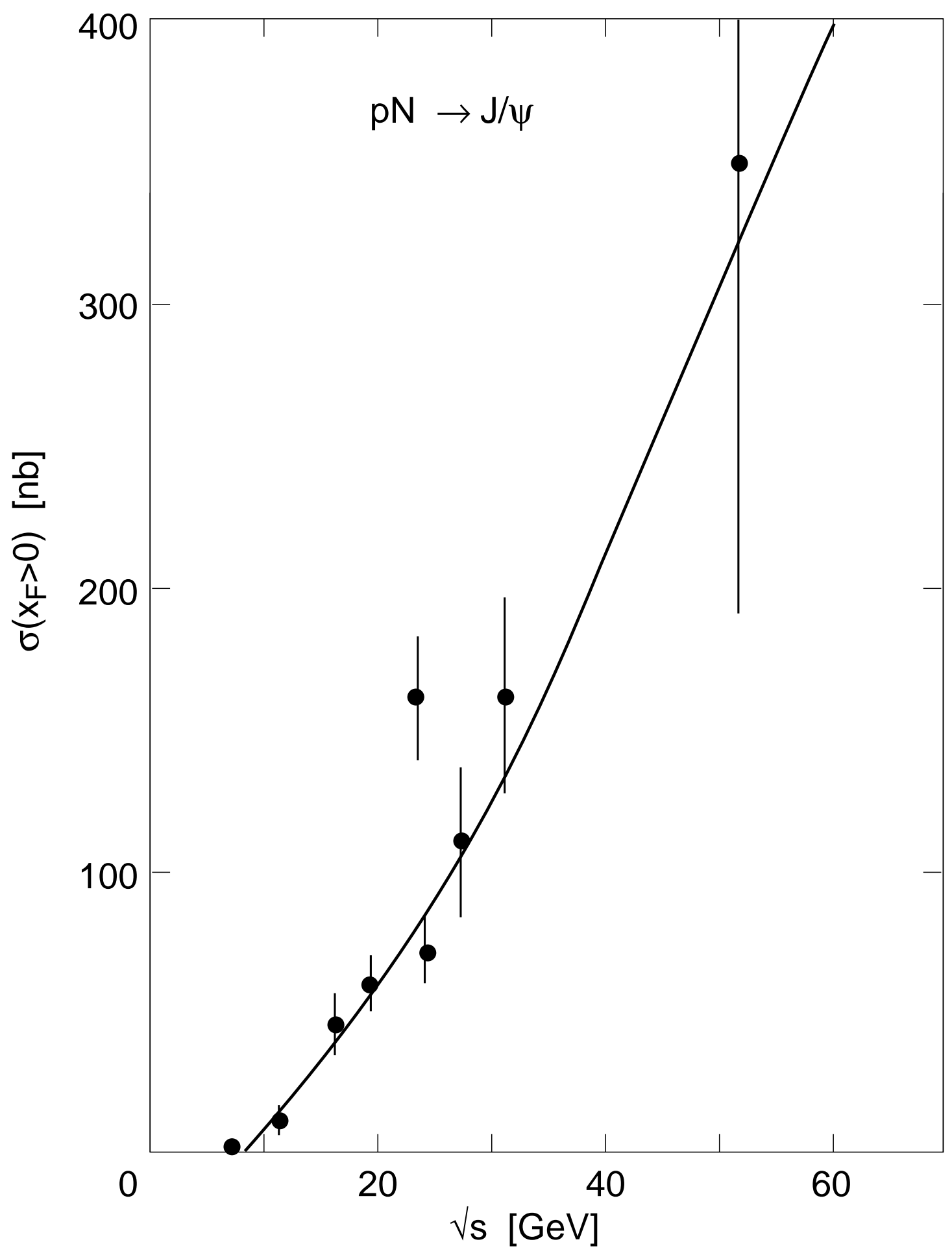

Figure 7: 


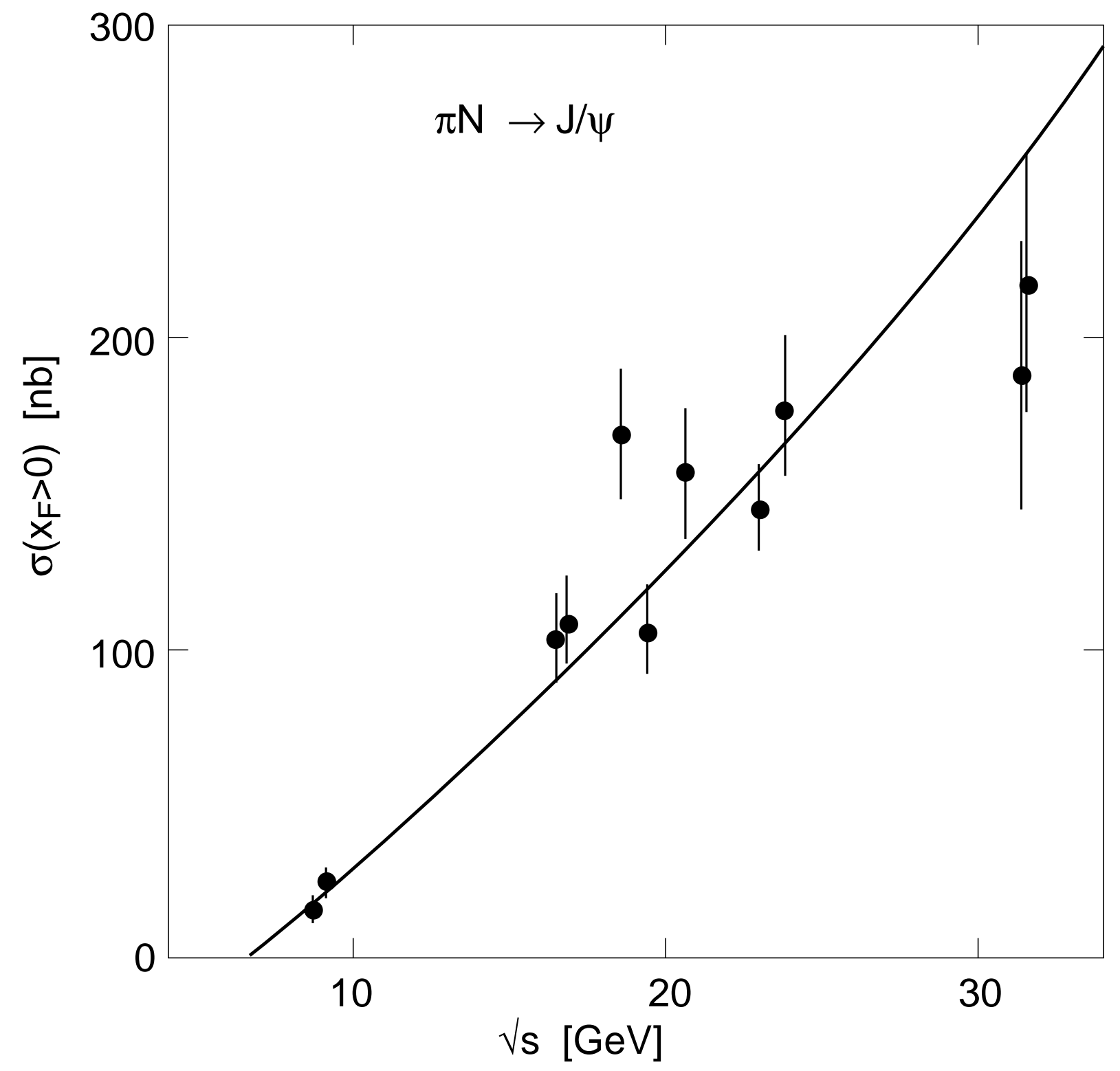

Figure 8: 


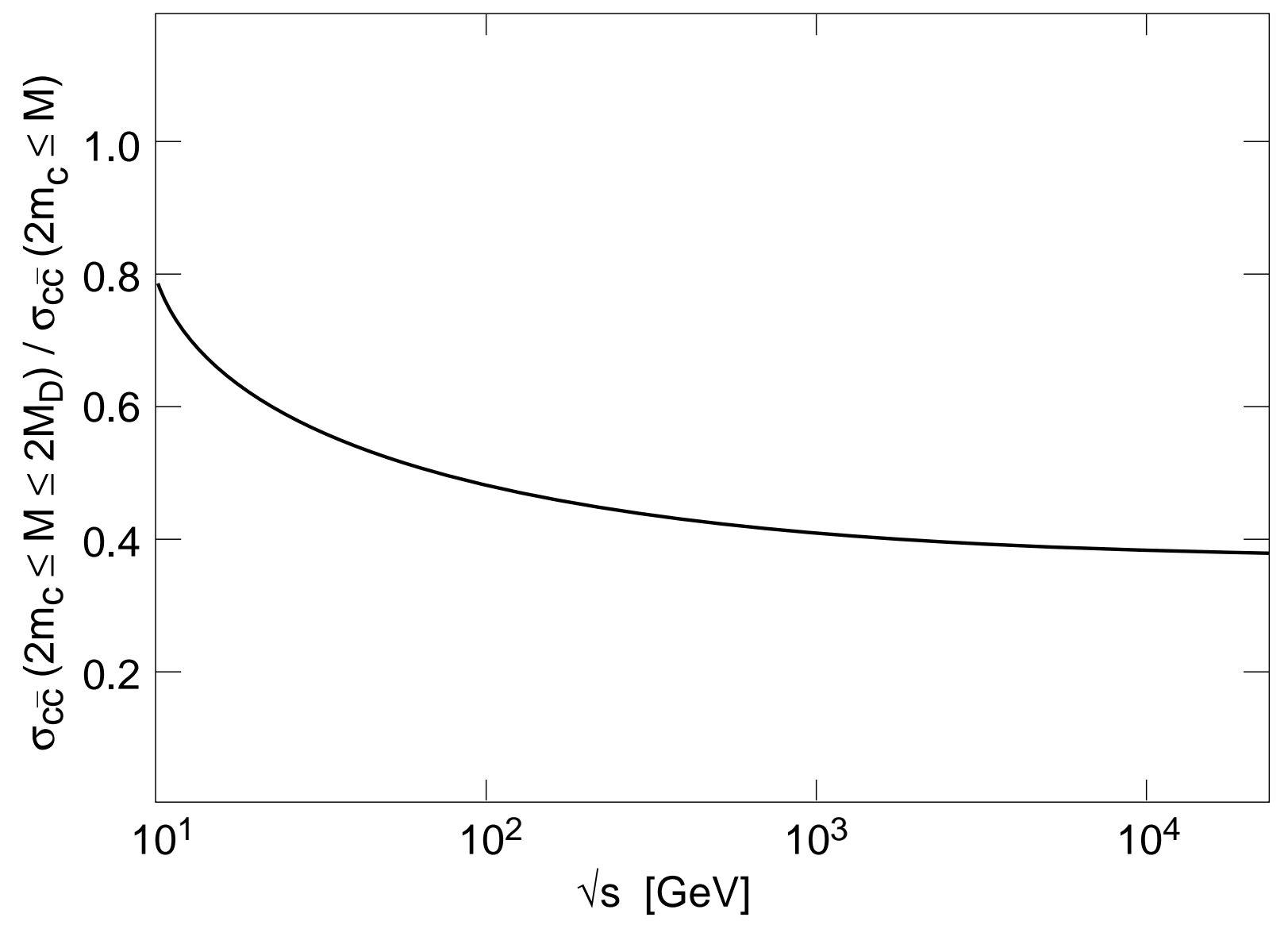

Figure 9: 

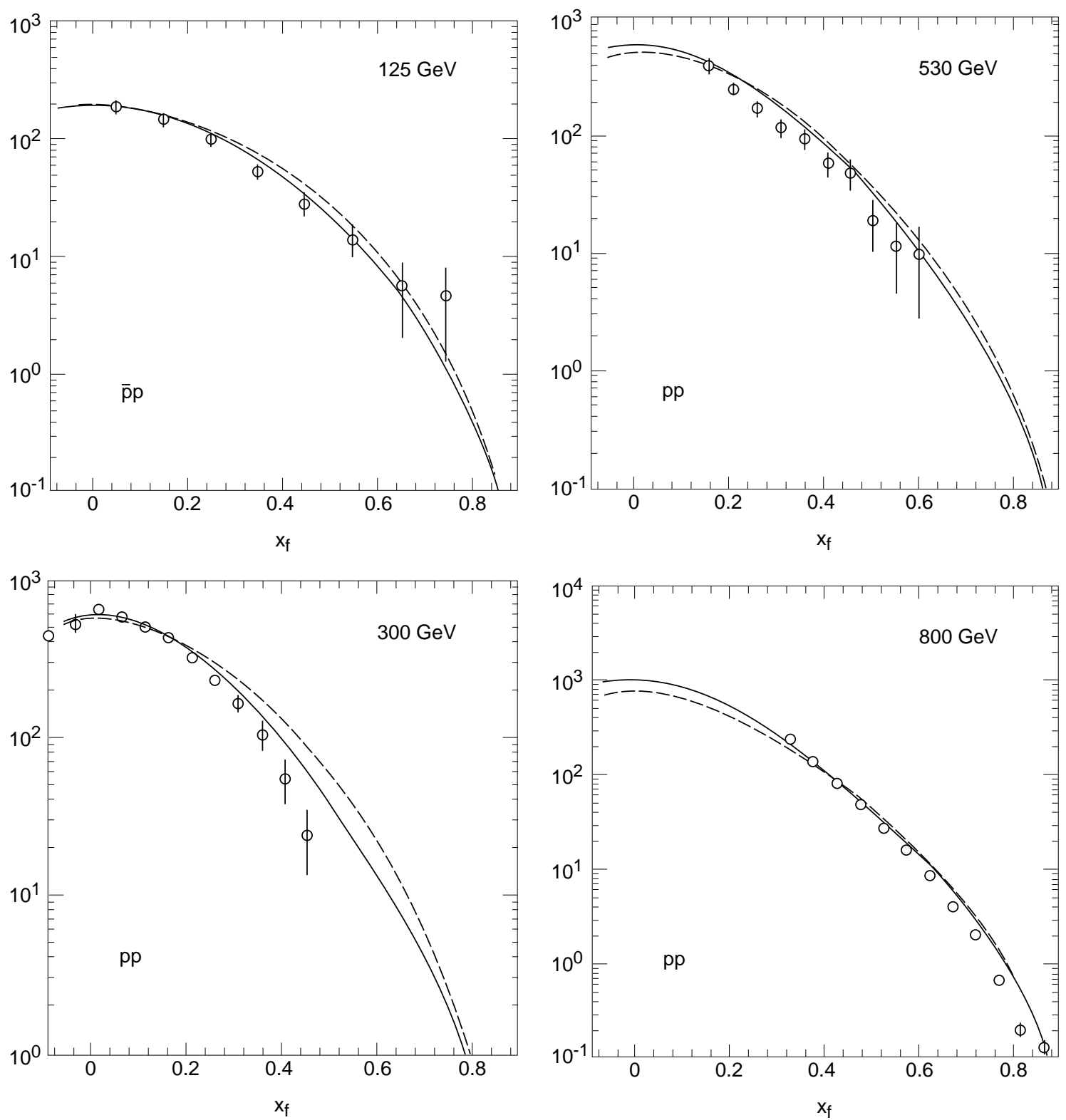

Figure 10a 

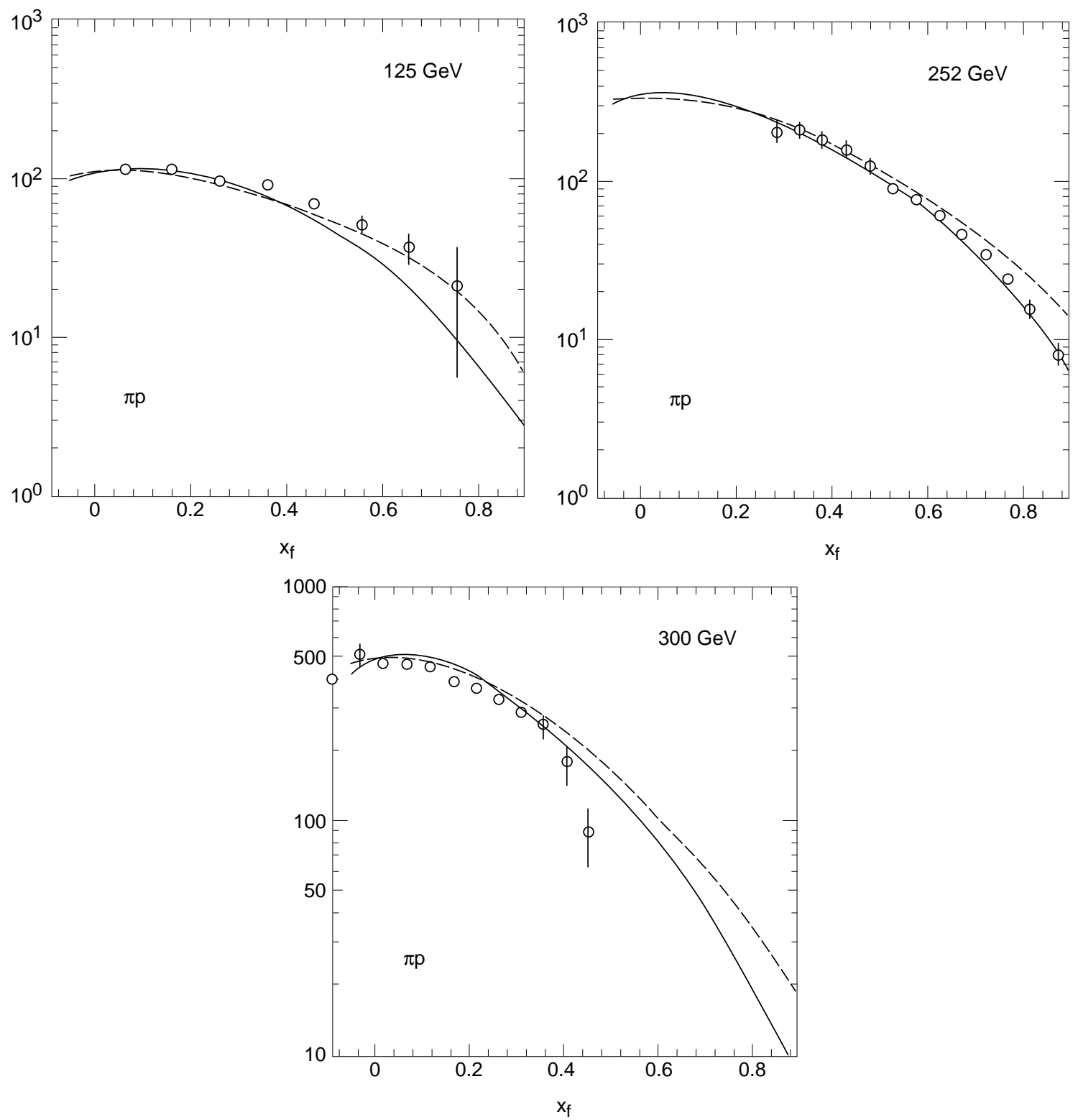

Figure 10b 


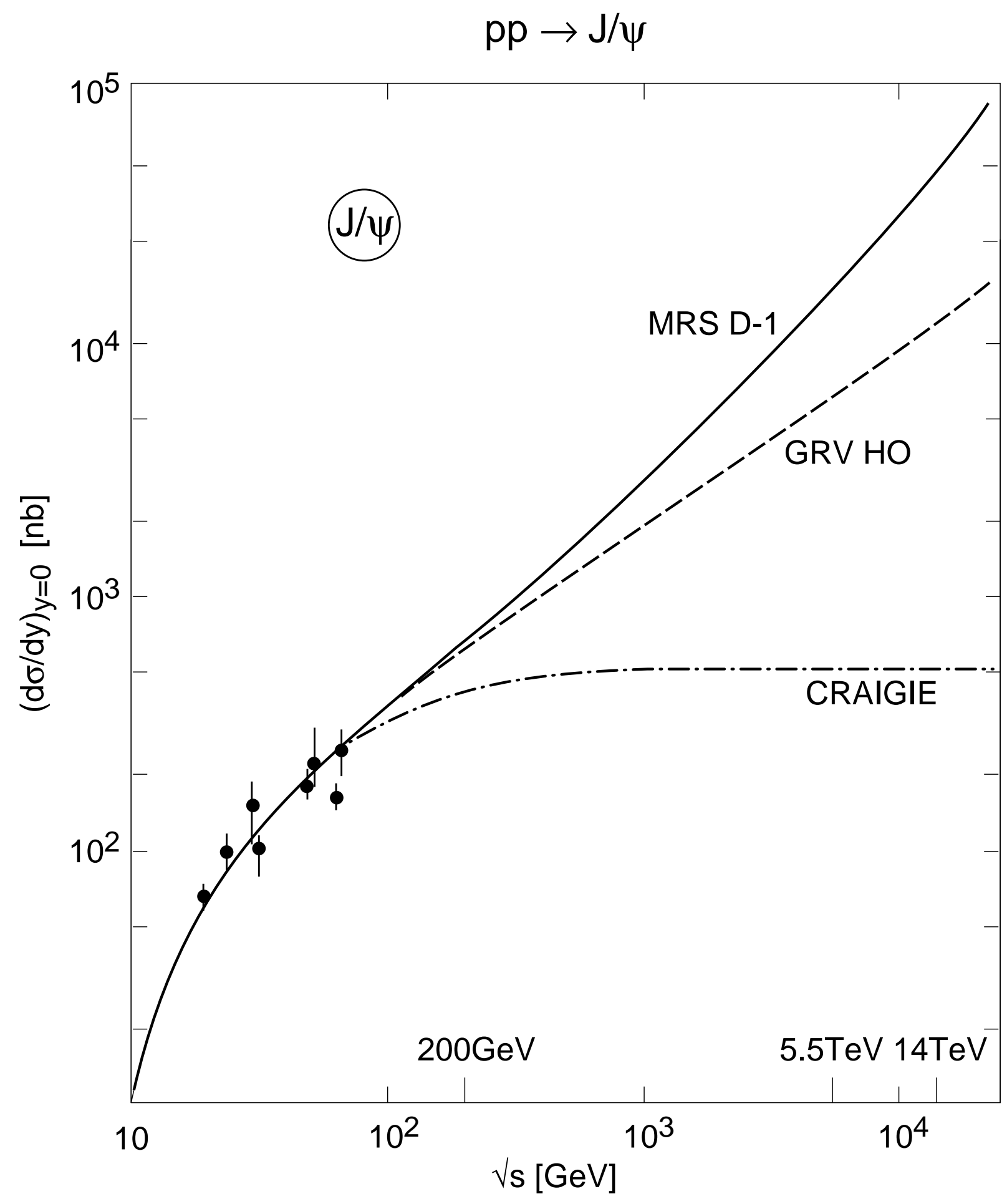

Figure 11: 


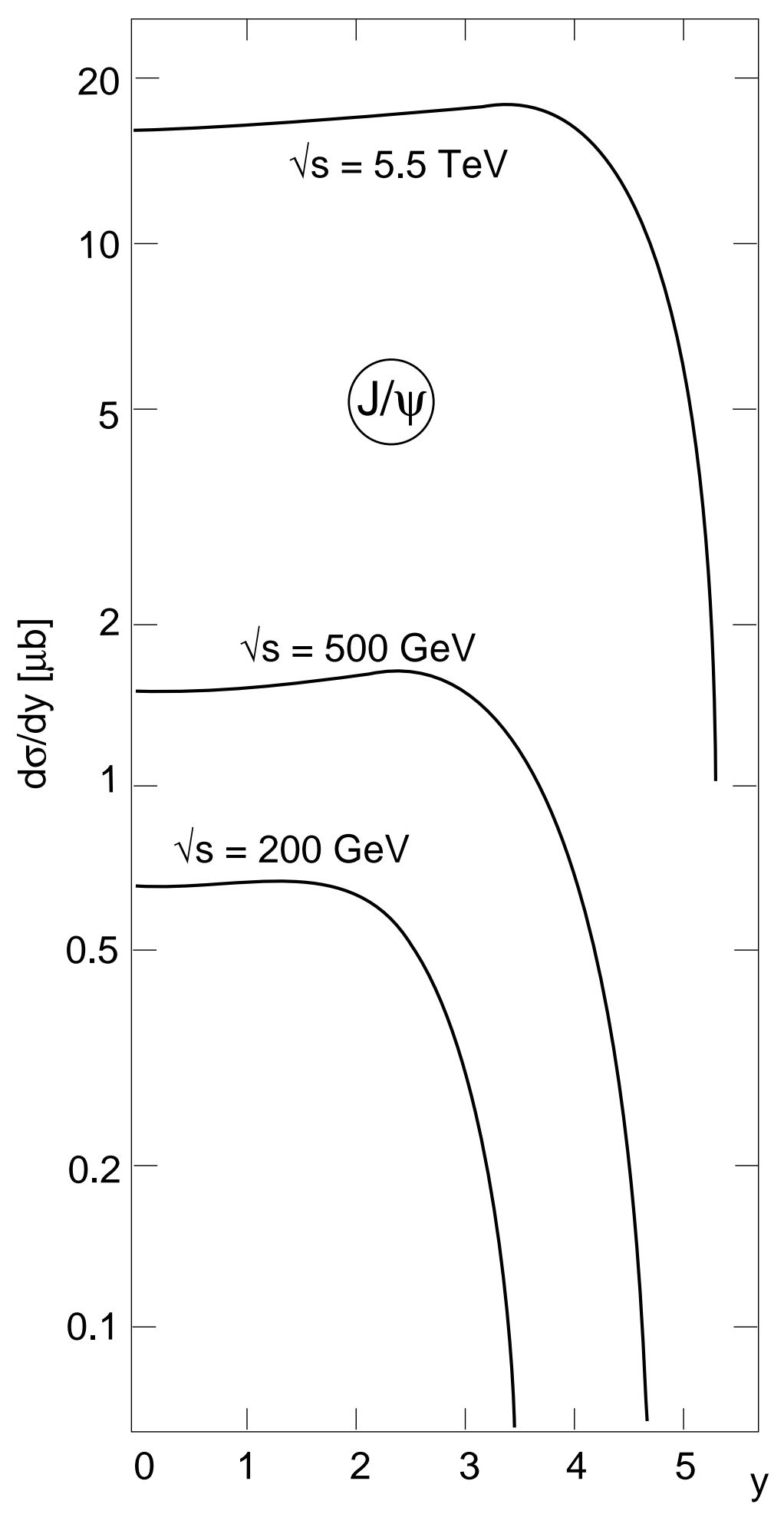

Figure 12: 


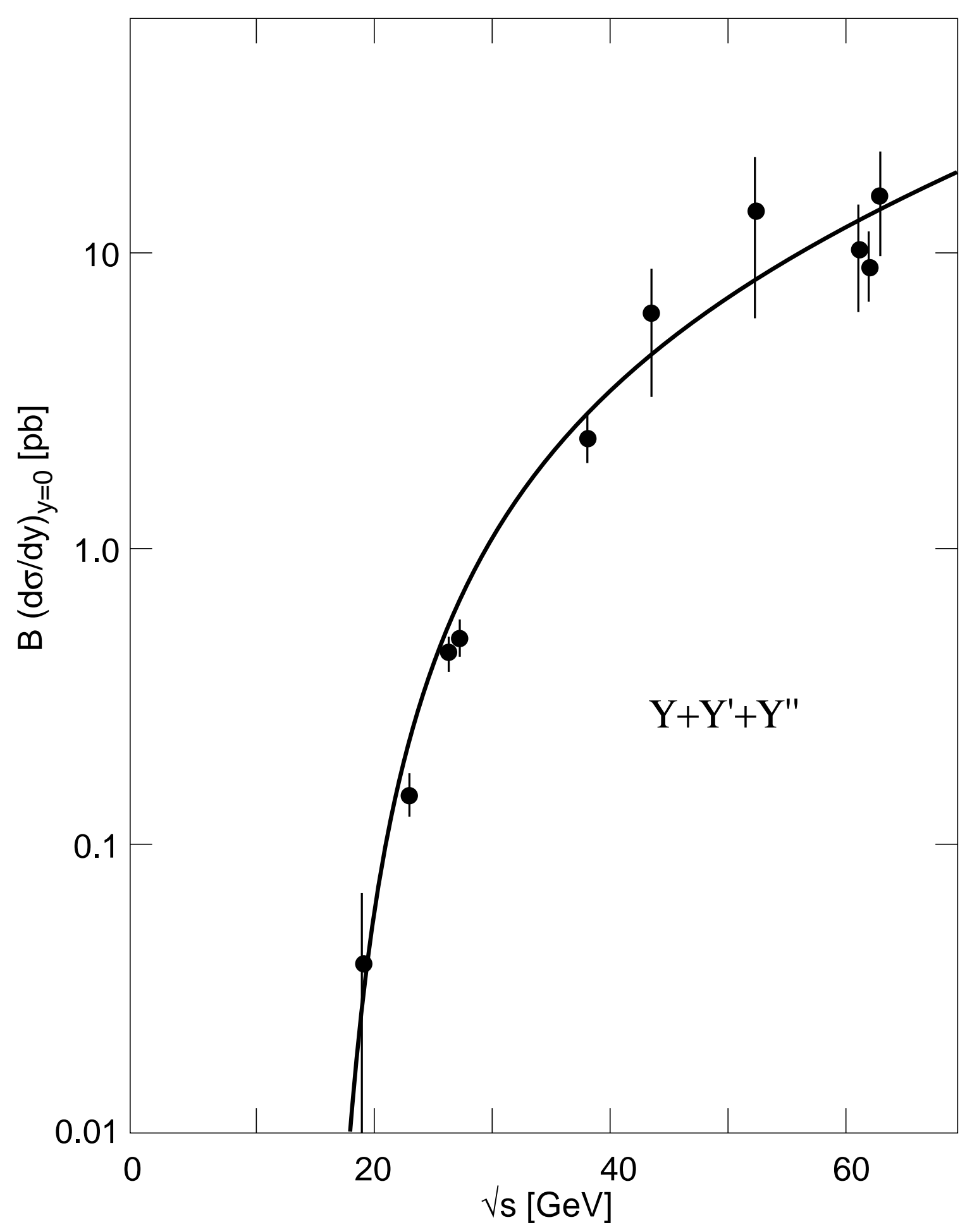

Figure 13: 


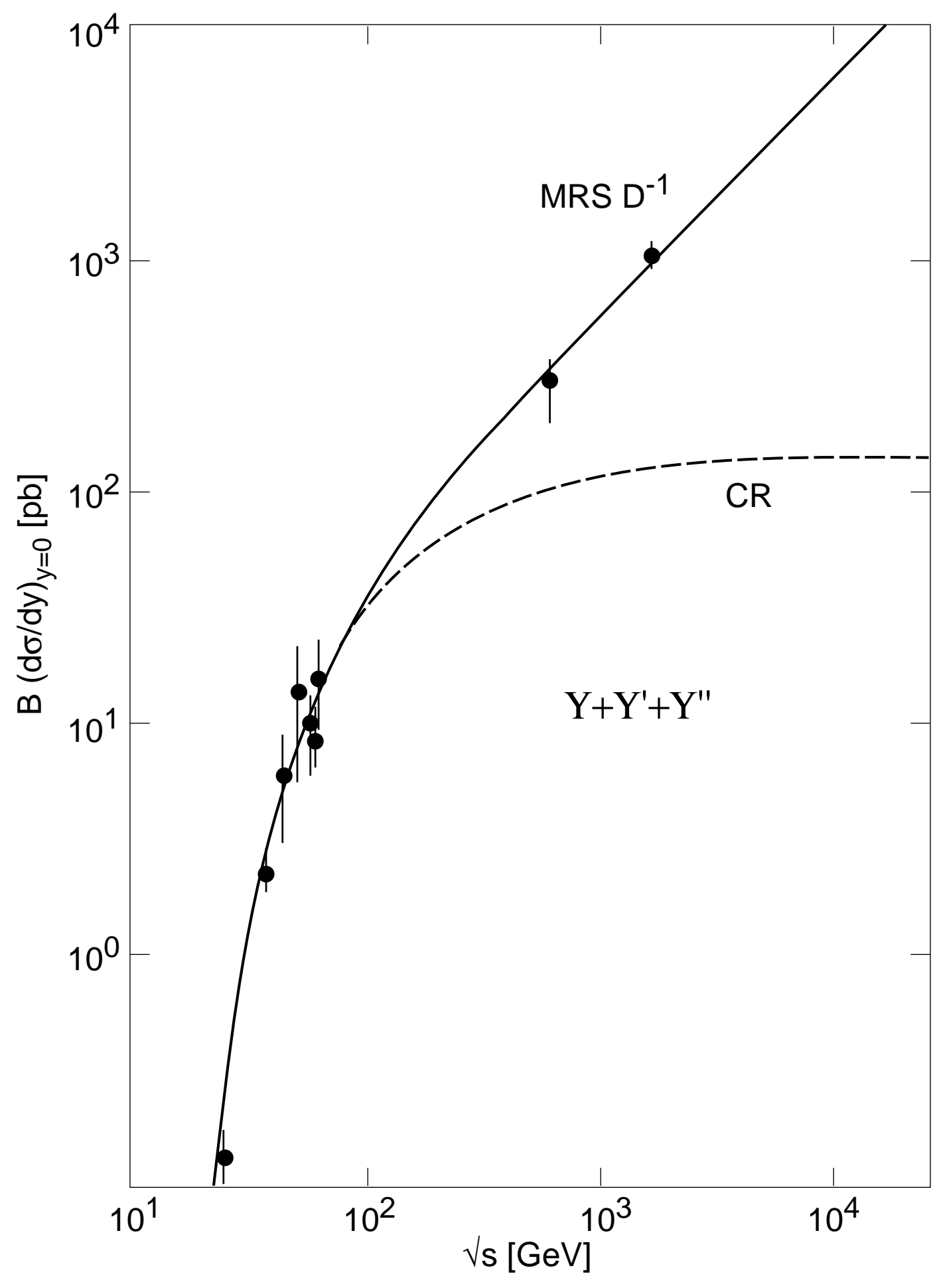

Figure 14: 


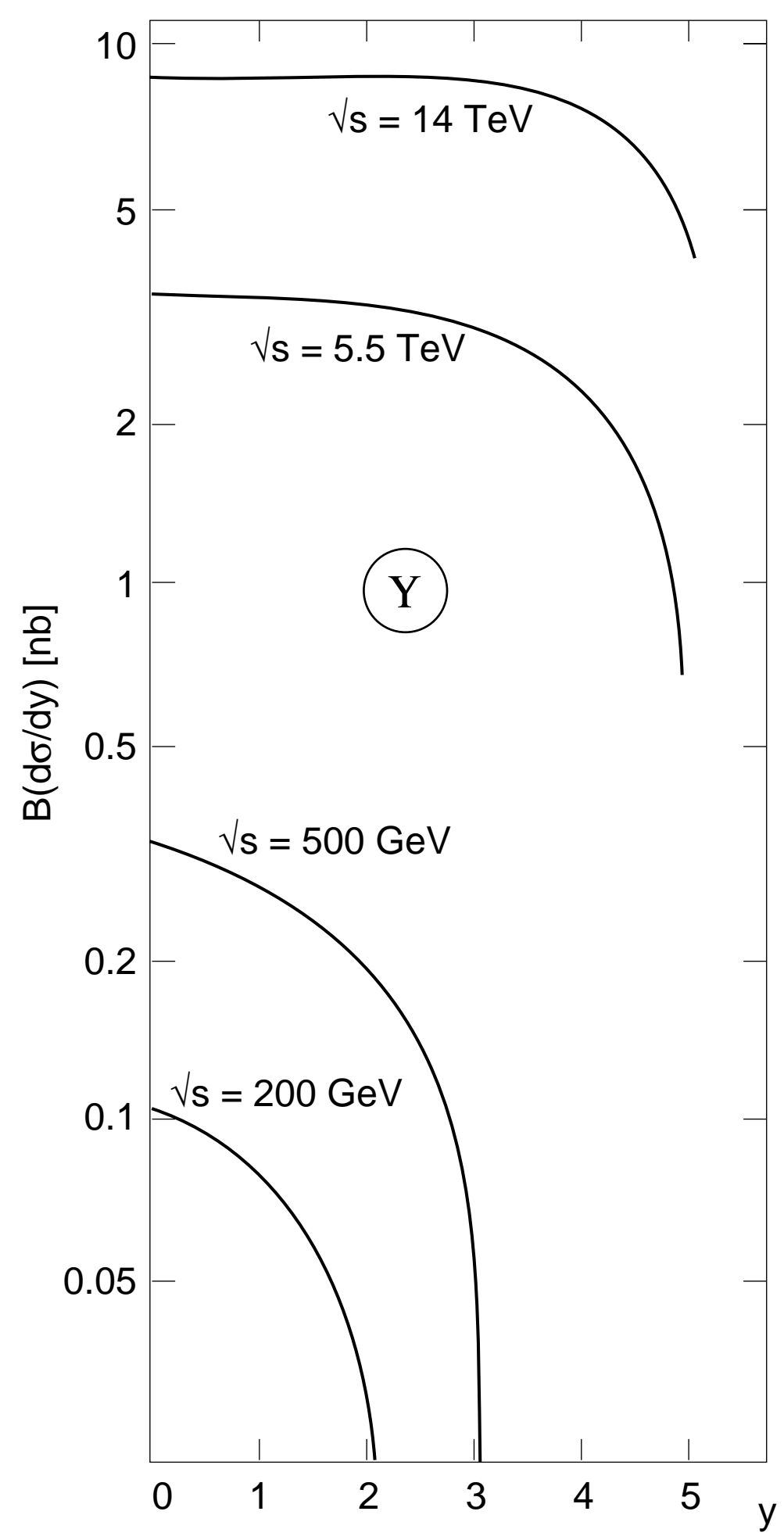

Figure 15: 

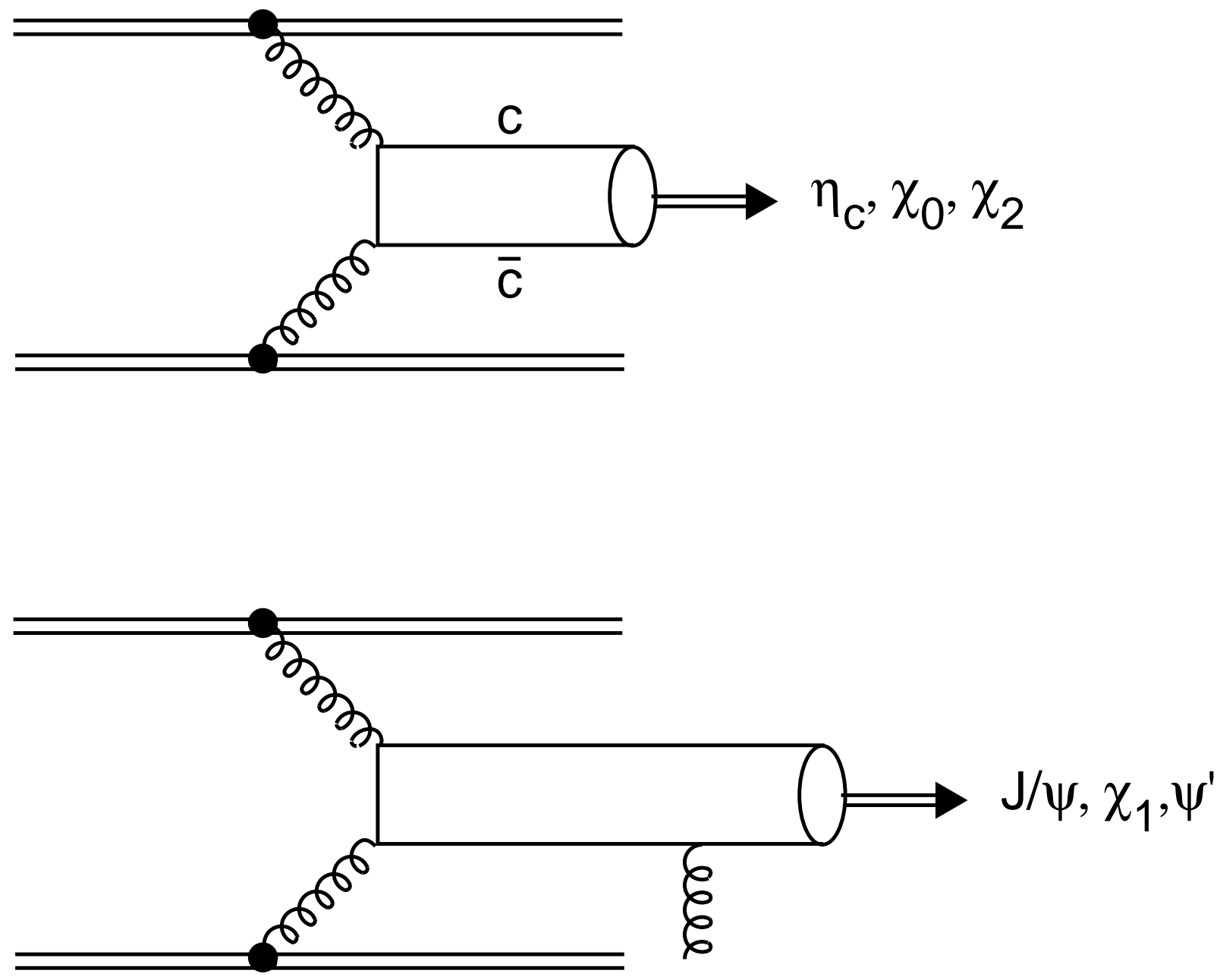

Figure 16: 